\title{
Dimensional symmetry breaking, information and fractal gravity in Cantorian space
}

\author{
M.S. El Naschie * \\ DAMTP, University of Cambridge Silver Street, Cambridge, CB3 9EW, UK
}

\begin{abstract}
We derive the exact expectation value and standard deviation for the dimensionality of Cantorian spacetime. Connections to time symmetry breaking, information and the generalization of complex numbers are considered. Finally a link between general relativity and Cantorian spacetime is proposed which amounts to the claim that fractalization is the origin of gravity. (C) 1998 Elsevier Science Ireland Ltd. All rights reserved.
\end{abstract}

Keywords: Symmetry breaking; Fractal spacetime; General relativity; Quantum gravity

\section{Introduction}

Why do we live in $3+1$ dimensions and what is the actual dimensionality of spacetime. Such questions may seem very unmodest or too 'philosophical' to be the subject of a serious scientific discourses. However, even if this attitude was ever correct in the past, it is surely now no more the case at least within modern theoretical particle physics. At present the situation is that apart of its epistemological importance, the question of the dimensionality of spacetime has an immediate bearing on vital calculation-technical aspects particularly in perturpative solution to problems in quantum field theory (Weinberg, 1996).

\footnotetext{
* Tel.: + 441223337733 .
}

To attempt to derive a probabilistic expectation value for space-time dimensionality is an idea which goes back to Wheeler (1964). A considerable time later, the present author found out that a Cantorian Fractal spacetime setting (El Naschie et al., 1995) provides an ideal frame work for Wheeler's proposal and that it ties logically very well with the superstring concept of dimensional compactifications (Svozil, 1987).

In this paper we first discuss the question of dimensionality within the Cantorian conception of space. Subsequently we will attempt to link our results to the question of time symmetry breaking which we regarded here to be a direct consequence of the existence of a finite expectation value for an otherwise infinite dimensional spacetime. Finally in the last part of this note we will discuss 'quantum' gravity in Cantorian spaces. 


\section{The Expectation value of the dimensionality of a Cantorian space}

We consider a space $\varepsilon$ which is supposed to resemble a kind of sigma field fractal. This field is assumed to be made up of an infinite number $(n=\infty)$ of elementary Cantor sets $\left(d_{\mathrm{c}}^{(0)}\right)^{n}$. The main equations are then easily derived using the basic intersection rule of probabilistic sets (El Naschie, 1994a, 1995).

$\Omega^{(n)}=\left(\Omega^{(1)}\right)^{n}$

where $n=1,2, \ldots$

Following the volume interpretation of the Hausdorff dimension (El Naschie, 1995), this equation can be rewritten as

$\Omega^{(n)}=\left(d_{\mathrm{c}}^{(0)}\right)^{n}=\left(\Omega^{(1)}\right)^{n}$

where $\left(d_{\mathrm{c}}^{(0)}\right)^{n}=d_{\mathrm{c}}^{(n)}$ and $d_{\mathrm{c}}^{(0)}$ is the kernel zero-dimensional set which will be regarded here as being a random variable (El Naschie and Prigogine, 1996).

To calculate an expectation value for $n$ we start by regarding every one of the infinitely many $(n=\infty)$ dimensions spanning $\varepsilon$ as having a weight $\left(d_{\mathrm{c}}^{(0)}\right)^{n}$ and consequently the weighted dimension is $w=n\left(d_{\mathrm{c}}^{(0)}\right)^{n}$. The moment of this dimension is consequently $M=n w$ and the centre of gravity is thus given by the expectation value (El Naschie and Prigogine, 1996).

$E(n)=S / A=\frac{\sum_{0}^{\infty} n^{2}\left(d_{\mathrm{c}}^{(0)}\right)^{n}}{\sum_{0}^{\infty} n\left(d_{\mathrm{c}}^{(0)}\right)^{n}}=\frac{\sum_{1}^{\infty} n^{2}\left(d_{\mathrm{c}}^{(0)}\right)^{n-1}}{\sum_{1}^{\infty} n\left(d_{\mathrm{c}}^{(0)}\right)^{n-1}}$

This way one finds immediately that

$E(n)=\langle n\rangle=\left(1+d_{\mathrm{c}}^{(0)}\right) /\left(1-d_{\mathrm{c}}^{(0)}\right)$

This is the first result of interest and we note that it is identical to the linear part of $E(n)$ as given by the continuous gamma distribution for 'Poissons arrival' $r=2$ (El Naschie and Prigogine, 1996).

While $\Omega^{(n)}$ is given first by Eq. (1) it can be also found from the intersection rule (El Naschie, 1994b)

$\Omega^{(n)}=(1 / m)\left(d_{\mathrm{c}}^{(0)}\right)$ where $m=d_{\mathrm{c}}^{(0)}$ is the Probabolistic Hausdorff dimension of finding a point in any of the $m$ dimensions consequently from Eqs. (1) and (5) one finds that

$\left(d_{\mathrm{c}}^{(0)}\right)^{n}=(1 / m)\left(d_{\mathrm{c}}^{(0)}\right)$

and therefore

$d_{\mathrm{c}}^{(n)}=\left(1 / d_{\mathrm{c}}^{(0)}\right)^{n-1}$

Equating Eqs. (4) and (7), the value of $n$ is fixed immediately by observing that

$\langle n\rangle=d_{\mathrm{c}}^{(n)}$

only if $n=4$ and $\left.d_{\mathrm{c}}^{(0)}=\phi=\sqrt{5}-1\right) / 2$ and consequently

$\langle n\rangle=d_{\mathrm{c}}^{(4)}=4+\phi^{3}=1 / \phi^{3}=1 /\left[d_{\mathrm{c}}^{(4)}-4\right]$

Note the formal similarity between $1 /\left[d_{c}^{(4)}-4\right]$ and Wilson-Fisher expansion and the simple poles $(d-4)^{-1}$ of dimensional regularization of quantum field theory (Weinberg, 1996).

Note also the remarkable continued fracture representation of $\langle n\rangle$ as given by Eq. (9) namely

$\langle n\rangle=d_{\mathrm{c}}^{(4)}=4+\phi^{3}=(4,4, \ldots)=(\overline{4})$

We look next at the expectation for $d_{\mathrm{c}}^{(n)}$. This is given by

$E\left(d_{\mathrm{c}}^{(n)}=\langle d\rangle=\sum_{n=1}^{\infty} n\left(d_{\mathrm{c}}^{(0)}\right)^{n-1} / \sum_{n=1}^{\infty}\left(d_{\mathrm{c}}^{(0)}\right)^{n}\right.$

Consequently we have

$$
\begin{aligned}
E(d) & =\langle d\rangle=\left[1 /\left(1-d_{\mathrm{c}}^{(0)}\right)^{2}\right] /\left[\frac{d_{\mathrm{c}}^{(0)}}{\left(1-d_{\mathrm{c}}^{(0)}\right)}\right] \\
& =\frac{1}{\left(1-d_{\mathrm{c}}^{(0)}\right) d_{\mathrm{c}}^{(0)}}
\end{aligned}
$$

Setting $d_{\mathrm{c}}^{(0)}=\phi$ in Eq. (12) we find the result of El Naschie (1994a), namely

$$
\langle d\rangle=\langle n\rangle=d_{\mathrm{c}}^{(4)}=4+\phi^{3}=(\overline{4})
$$

Next we look closely at $d_{\mathrm{c}}^{(0)}$ as related to $\langle d\rangle$. Setting $\langle d\rangle=4$, one finds

$\left(1-d_{\mathrm{c}}^{(0)}\right) d_{\mathrm{c}}^{(0)}=1 / 4=1 /\langle d\rangle$

or

$\left\langle d_{\mathrm{c}}^{(0)}\right\rangle=+1 / 2$ 
In a similar way it can be shown that in $\varepsilon$, the mean, median, minimum and four-dimensional kernel $\left\langle d_{\mathrm{c}}^{(0)}\right\rangle$ (for $\langle d\rangle=4$ ) are all equal

$\left\langle d_{\mathrm{c}}^{(0)}\right\rangle=d_{\mathrm{cmin}}^{(0)}=d_{\mathrm{cmed}}^{(0)}=1 / 2$

It is interesting to note now that for this value we have the anomalous situation that

$\langle n\rangle=3 ; \quad\langle d\rangle=4$

and consequently

$\langle n\rangle\langle\langle d\rangle$

Further more it should be noted that for $d_{\mathrm{c}}^{(0)}=1 / 2$ we have ${ }^{1}$

$d_{\mathrm{c}}^{(4)}=\left(1 / \frac{1}{2}\right)^{3}=8$

\section{The question of $3+1$ dimension}

As we have just seen, the space $\varepsilon$ with $n=\infty$ has some surprising properties. First, the expectation value $\langle n\rangle=\left(1+d_{\mathrm{c}}^{(0)}\right) /\left(1-d_{\mathrm{c}}^{(0)}\right)$ is given by $4+\phi^{3}$ for space filling dynamic (Sagan, 1994). This is, of course, a natural enough condition for our space.

Second, the expectation value for the Hausdorff dimension of $\varepsilon$, namely $\langle d\rangle$, is equal to $\langle n\rangle=d_{\mathrm{c}}^{(n)}$ only if $d_{\mathrm{c}}^{(0)}=\phi$, the Golden Mean, and also $n=4$. Thus four dimensionality is singled out by the requirement that $\varepsilon$ is space filling. The average value of $d_{\mathrm{c}}^{(0)}$ which lays exactly between a zero Menger-Urysohn topological dimension Cantor set and the continuous line $d_{\mathrm{c}}^{(1)}=1$, according to Eq. (8) is thus given by $\left\langle d_{\mathrm{c}}^{(0)}\right\rangle=1 / 2$ in full agreement with Eq. (16). It is also clear from the previous analysis that the Eq. (7) introduces to the Menger-Urysohn dimensional system the notion of a degree of emptiness (without affecting the axiomatic structure of ZF set theory) because $n=-1$ corresponds to the empty set while in our case only $n=-\infty$ represents a truly empty set (El Naschie, 1994c).

As mentioned earlier the possibility of consider-

\footnotetext{
${ }^{1}$ However, noting that $\langle n\rangle=3$ for $d_{\mathrm{c}}^{(0)}=1 / 2$, we find that $\left\langle d_{\mathrm{c}}^{(n)}\right\rangle=(1 /(1 / 2))^{3-1}=4$.
}

ing $\varepsilon$ to model micro space-time was addressed by the author in several previous publications. Such 'physical' interpretation of $\varepsilon$ as a vacuum may lead to certain speculation about perception and brain function (Penrose, 1990, 1994). Nevertheless, the four dimensionality $n=4$ and $\langle n\rangle=$ $\langle d\rangle=(\overline{4})$ is indeed striking. It may therefore be possible to try to stretch the idea further still and ask why $3+1$ and not simply 4 dimensions is the dimensionality of our real space (El Naschie et al., 1996a).

Now if we could draw on the quite speculative idea that our mental structure permits an awareness of $\langle d\rangle$ but a direct physical access only to $\langle n\rangle$ or $n$ then we could give the following explanation. Although $\left\langle d_{\mathrm{c}}^{(0)}\right\rangle=1 / 2$ we have $n=3$ but $\langle d\rangle=4$. The extra dimension of $\langle d\rangle$ will therefore be felt but cannot be directly measured, or in fact 'seen'. This 'number theoretical' argument is once more reinforced by Eq. (7) because $d_{\mathrm{c}}^{(3)}=4$ for $d_{\mathrm{c}}^{(0)}=1 / 2$. In other words, for $d_{\mathrm{c}}^{(0)}=1 / 2$ we have $n=\langle n\rangle=3$ but $d_{\mathrm{c}}^{(3)}=\langle d\rangle=4$. We are quite aware that this is a somewhat daring hypothesis but nevertheless wonder if it would not be worth pursuing a little further (El Naschie et al., 1996b).

\section{Symmetry breaking in Cantorian space, the arrow of time and information}

From the preceding discussion we can infer that Cantorian space-time $\varepsilon$ is fully spacialized infinitedimensional $(n=\infty)$ quasi-random space made up of the intersections of an infinite number of space-filling Cantor sets which has the structure of a semi-group-like (non-invertible) iterated function system. However, and against naive expectations we have seen that the 'weighted' gamma distribution mean value of the topological dimension of $\varepsilon$ coincides exactly with the dimensionality of the real space-time of our experience.

Since all of these results were obtained using a gamma distribution function ${ }^{2}$ it follows that they are intimately connected to the thermodynamical statistics of Maxwell-Boltzmann (El Naschie,

\footnotetext{
${ }^{2}$ This is the same distribution used to derive the Plack formula for black body radiation.
} 
1996). The most striking point about $\varepsilon$ remains, however, that it picks up four dimensionality as an expectation value which happens to be our dimensional reality. A recent, similar, purely mathematical derivation is due to Hemion (El Naschie, 1996) and Al Athel (El Naschie et al., 1996b). This shows that four is the dominant value while $n>4$ are assigned a very small probability. Since we are regarding the entire universe, we may be justified in viewing the situation in a different way, similar to that of string theory, and regard all dimensions $n>4$ as being too small and compactified to be observed directly (El Naschie et al., 1995; Svozil, 1987). The connection between fractal spacetime dimension and compactification was pointed out in general terms by Svozil (1987).

In turn, this result could be interpreted as a kind of sudden dimensionality reduction or dimensional symmetry breaking in the informational content of $\varepsilon$ which takes place as we move towards a macro level. In other words, the four dimensionality starts somewhere along the way from the subquantum world towards the semiclassical and then the macro world. This transition takes place near the de Broglie length scale (El Naschie et al., 1996a,b) at which information from the infinitely many extra dimension has a diminishing and extremely small effect on the motion of classical objects. ${ }^{3}$

The importance of this chain of thought now lies in the possibility of immediately deducing from them the following conclusion. While in principle quantum and subquantum particles can travel back in fractal-Cantorian time 'history', macro and quasi macro particles could obviously not do that, not even in principle, except in the sense of conservative classical mechanics which is of course trivial. The obvious reason for this is that because a classical particle is sufficiently 'large' to persist in the expectation space $(\langle n\rangle=4)$, it is now behind a Cantorian transfinite wall acting as an 'informational' barrier so to speak, preventing it from going back in its own time 'history' unless it dissolves in much smaller quantum or subquantum particles which can travel in all the infinite dimensions of $\varepsilon$ without encountering the preceding dimensional 'information' difficulty.

\footnotetext{
${ }^{3}$ This is very similar to a Bethe lattic.
}

It is clear that the Huasdorff dimension, being an intermediate measure for volume and dimension, also plays an important role here as a link between dimension and information. Should we now conjecture a relation between irrationality and content of information then, because $\phi=(\sqrt{5}-1) / 2$ is the most irrational number, the informational content of $d_{\mathrm{c}}^{(4)}(\varepsilon)$ must be the largest possible. ${ }^{4}$

\section{Generalization of numbers and Cantorian spaces}

It is a well known mathematical theorem, Frobenius theorem, which states that the four dimensional quartrions are the only generalization of real numbers. This is of course Hamilton's generalization of complex numbers which cannot be done in three or more than four dimensions. Together with Donaldson's discovery of fake $R^{4}$ this seems to stress the importance of $d_{\mathrm{c}}^{(4)}$ of our Cantorian space-time.

However it is also well known that if we give up not only commutativity but also associativity then complex numbers may be generalized to eight dimensional structures called octanions. Further generalization to higher dimensions is no longer 'possible'. It is tempting now to see some analogy between octions and our $d_{\mathrm{c}}^{(8)}$ which are supposed to be related to eightfold symmetry, Kepler's conjecture as well as quarks and SU(3) (El Naschie, $1995,1996)$. Note also that $d_{\mathrm{c}}^{(4)}=8$ for $d_{\mathrm{c}}^{(0)}=1 / 2$ as well as that the standard deviation leads to $\langle n\rangle_{\max }=8$.

To sum up, we could say that many of the contradictions in quantum physics stem from the fact that while the universe is infinite dimensional all our measurements are taken not in $\varepsilon$ but in the expectation value of $\varepsilon$ for which we have $\langle n\rangle=4$. We are three-dimensional and move in three dimensions, the fourth dimension is already a threshold since we feel the time dimension but

\footnotetext{
${ }^{4}$ There are interesting connections between $\varepsilon$ and 4 manifolds as well as Noncommutative geometry. For instance the indix $\tau=b^{4}-b^{-}$could be also written as $\tau=\left(b^{+}\right)\left(b^{-}\right)$when setting $b^{+}=\operatorname{Dim}$ ker $\varepsilon$ and $b^{-}=$Dim Coker $\varepsilon$. Consequently for $\varepsilon$ one finds $b^{+}=\phi$ and $b^{-}=(1-\phi)$. That means $1 / \tau=1 /$ $\phi^{3}=(4,4, \ldots)$. (El Naschie, 1998).
} 
cannot see it. The fifth dimension manifest it 'self only indirectly may be as spin $1 / 2$ particle. The next threshold could be the eight dimensional space which is related to quarks and may be to the limit of any computation using anything like numbers which are suitable to tackle this space. It is also important and extremely interesting to note at this point that our Cantorian-fractal concept of quantum spacetime may be closely related to the so called Biedenharn-Horwitz conjecture on time space (Biedenharn and Horwitz, 1978) in which there exists no apriori scale for a single path and may therefore be arbitrarily subdivided leading to a structuring of spacetime and to the introduction of a 'meta-time'. Some of the ideas presented by Augenstein maybe also very useful in the present context (Augenstein, 1996).

\section{6. 'Quantum' gravity and Cantorian spacetime}

The first departure from Newton's classical concept of an absolute time was of course Einstein's special theory of relativity (SR) (Weyl, 1952). In this theory time did not depend only on the initial and final conditions, but also on the 'path' itself. This is naturally a consequence of fusing space and time to spacetime. Thus in special relativity time has lost some of its absoluteness and invariant properties. Spacetime of SR is thus real but nonmaterial and independent of anything else exactly as in Newton's physics. By contrast, the idea of time in general relativity (GR) is a complete and radical departure from Newton (Weyl, 1952; Thorne, 1994; Ciufolini and Wheeler, 1995; Sexl and Urbantke, 1995). Time here is not only 'path' dependent but also no longer the same everywhere and is affected by matter. We say usually that gravitational field causes time to 'fluctuate' in space as shown by the 'gravitational redsheft', but it is in fact a far more radical situation than that Unruh (1995). Within Einstein's general theory, gravity itself could be conceived as a derivative of time and a direct consequence of its' fluctuation in space. Gravity is a phenomenon caused by time 'flowing' with different 'speeds'. It is an uneven 'flow' of time from place to place (Unruh, 1995). Seen that way, matter and gravity do not just affect time and cause watches to run differently but it is 'in a more direct sense' a derivative of time.

From the preceding discussion it is evident that the Cantorian-fractal conception of spacetime may have many things to offer in the current efforts towards a reconciliation between quantum mechanics (Q.M.) and G.R. Cantorian spacetime and particularly fractal time is by definition different from 'point' to 'point'. It flows nonuniformly and is intrinsicly and irreducibly probabilistic in nature. Spacetime and consequently as we have just reasoned gravity, could be converted to pure statistical geometrical consideration in the sense of $\mathrm{K}$. Menger and the modern development of his and G. Cantor's ideas.

Through the systematic use of Cantorian spacetime interpretation we might be able indeed, at least within an abstract model to give up momentarily the idea of gravity as a force and replace it by the idea that it is related to a nonvanishing component or an average deviation of fractal time from linear uniform time which is nothing but an approximation of the 'real' micro spacetime within the resolution of classical physics (El Naschie et al., 1996b).

It is also well known that within GR not only time is involved in causing gravity, but also space. This is most clear from Friedman's solution of Einstein's equations which led eventually to the big bang theory of the creation of spacetime.

Within Cantorian spacetime in which time is completely specialized the same conclusion can be drawn. Now if we regards the Cantorian-fractal theory of spacetime as basically and simply a theory which takes R. Feynman's path integral formulation seriously and literally to be a physical reality as expressed recently (by G. Hemion, 1996, Private communication), then we have here a direct and extremely useful connection to Wheeler-Dewitt equation, basically a Schroedinger equation for the entire universe as well as S. Hawking's work (Thorne, 1994; Ciufolini and Wheeler, 1995; Sexl and Urbantke, 1995). This view may then reconcile Q.M. and G.R. which seems to force on us the idea that we must abandon the Hilbert space formulation and go over to may be I. Prigogine's RiggedGelfand formulation or our Cantorian-fractal space setting (El Naschie, 1996).

It is extremely interesting to note that recently, Unruh in an inspiring and very readable article has 
presented ideas similar to the present one (Unruh, 1995), but without invoking Cantorian fractal spacetime arguments.

In conclusion we may note that the view that gravity is caused by the 'fractal' fluctuation of time is not as radical a proposal as it may seems at first sight. It is well established fact in molecular physics for instance that the attractive van der Waal's force is explained in terms of the dipole moments induced by the fluctuation in the molecules distribution. This led R. Feynman to speculate in his acclaimed lectures on Gravitation that gravity may be an attraction due to similar fluctuation of 'something' (Feynman, 1995) the present note proposes that taking Einstein's gravity and Ord's fractal time together may be a way to identify this 'something' with Cantorian spacetime (El Naschie, 1997) in this sense we are proposing that fractalization is the origin of gravity. Within this theory, gravity itself remains 'almost' classical. ${ }^{5}$

\section{Acknowledgements}

I am extremely indebted to G. Ord for discussion, stimulation and many critical remarks. I am also indebted to G. Joshi of the School of Physics, University of Melbourne for drawing my attention to the Biedenharn-Horwitz conjecture. Finally, I thank G. Hamion of the Department of Math, Bielefield (Germany) for stimulating correspondence.

\section{References}

Augenstein, B.W., 1996. Links between physics and set theory. Chaos, Solitons and Fractals 7 (11), 1761-1798.

Biedenharn, L., Horwitz, L., 1978. Quantum theory and exceptional gauge groups. Proc. 2nd Johns Hopkins Workshop, 21st April.

\footnotetext{
${ }^{5}$ In fact $\left\langle d_{\mathrm{c}}^{(0)}\right\rangle=1 / 2$ means $d_{\mathrm{c}}^{(2)}=d_{\mathrm{s}}=2$ and following Born "interpretation" it can be shown that $d_{\mathrm{H}}=\left\langle d_{\mathrm{s}} / d_{\mathrm{s}}\right\rangle=2 \otimes 2=4$. The fact that $d_{\mathrm{H}}$ could be also found from $d_{\mathrm{H}}=2 \oplus 2=4$ is relevant to the two-slit experiment, quantum gravity and squeezed quantum states. (El Naschie, 1998)
}

Ciufolini, I., Wheeler, J.A., 1995. Gravitation and Inertia, Princeton, NJ.

El Naschie, M.S., Prigogine, I., 1996. Time symmetry breaking in classical and quantum mechanics. Chaos, Solitons and Fractals 7 (4) (Special Issue).

El Naschie, M.S., Rössler, O.E., Prigogine, I. 1995. Quantum Mechanics, Diffusion and Chaotic Fractals. Elsevier, Oxford.

El Naschie, M.S., Rössler, O.E., Ord, G., 1996a. Information and diffusion in quantum physics. Chaos, Solitons and Fractals 7 (5) (Special Issue).

El Naschie, M.S., Nottale, L., Al Athel, S., Ord, G., 1996b. Fractal space-time and Cantorian geometry in quantum mechanics. Chaos, Solitons and Fractals 7 (6) (Special Issue).

El Naschie, M.S., 1994a. Dimensions and Cantor spectra. Chaos, Solitons and Fractals 4, 2121-2132.

El Naschie, M.S., 1994b. Is quantum space a random Cantor set with a Golden Mean dimension at the core? Chaos, Solitons and Fractals 4, 177-179.

El Naschie, M.S., 1994c. On certain 'empty' Cantor sets and their dimensions. Chaos, Solitons and Fractals 4, 293-296.

El Naschie, M.S., 1995. Banach-Tarski theorem and Cantorian micro space-time. Chaos, Solitons and Fractals 5, 15031508.

El Naschie, M.S., 1996. Wick rotation, Cantorian spaces and the complex arrow of time in quantum physics. Chaos, Solitons and Fractals 7 (9), 1501-1506.

El Naschie, M.S., 1997. A Note on quantum gravity and Cantorian spacetime. Chaos, Solitons and Fractals 8 (1), 131-133.

El Naschie, M.S. 1998. On the irreducibility of spacial ambiguity in quantum physics, C, S\&F 9, in press.

Feynman, R., 1995. In: Hatfield, B. (Ed.), Lectures on Gravitation. Addison-Wesley, New York.

Penrose, R., 1990. The Emperor's New Mind. Oxford University Press, New York.

Penrose, R., 1994. Shadows of the Mind. Oxford University Press, New York.

Sagan, H., 1994. Space-Filling Curves, Springer Berlin.

Sexl, R.U., Urbantke, H.K., 1995. Gravitation and Kosmologie. Spektrum, Berlin.

Svozil, K., 1987. Quantum field theory on fractal space-time: a new regularisation method. J. Phys. A. Math. Gen. 20, $3861-3875$.

Thorne, K.S., 1994. Black Holes and Time Warps. Picador, London.

Unruh, W., 1995. Time, gravity and quantum mechanics. In 'Time's Arrows today'. Cambridge University Press, Cambridge, pp. $23-65$.

Weinberg, S., 1996. The Quantum Theory of Fields, vol. II. Cambridge University Press, Cambridge.

Weyl, H., 1952. Space-Time-Matter. Dover, New York.

Wheeler, J.A., 1964. Geodynamics and the issue of the final state. In: Dewitt, C., Dewitt, B. (Eds.), Relativity, Groups and Topology. Gordon and Breach, New York. 